\title{
A Survey of Soil-Transmitted Helminths Infections and Schistosomiasis mansoni among School Children in Libo-Kemkem District, Northwest Ethiopia: Cross Sectional Study
}

\author{
Tesfahun Addisu ${ }^{1}$, Achenef Asmamaw ${ }^{2}$ \\ ${ }^{1}$ Department of Medical Laboratory Science, College of Health Science, Bahir Dar Health Science College, Bahir Dar, Ethiopia \\ ${ }^{2}$ Department of Obstetrics and Gynaecology, PAN African University, University of Ibadan, Ibadan, Nigeria
}

Email address:

tesfahunhimi@gmail.com (A. Tesfahun), ashua2014@gmail.com (A. Asmamaw)

\section{To cite this article:}

Tesfahun Addisu, Achenef Asmamaw. A Survey of Soil-Transmitted Helminths Infections and Schistosomiasis mansoni among School Children in Libo-Kemkem District, Northwest Ethiopia: Cross Sectional Study. American Journal of Health Research.

Vol. 3, No. 2, 2015, pp. 57-62. doi: 10.11648/j.ajhr.20150302.12

\begin{abstract}
The burden of disease caused by infection with schistosomiasis and soil-transmitted helminthes remains enormous. It is widely recognized that school children carry the heaviest burden of morbidity. Apart from the morbidity associated with acute infections, those who are chronically infected are often physically and intellectually compromised by anaemia, leading to attention deficits, learning disabilities, school absenteeism and higher dropout rates. Taking this into consideration, the aim of this study was to investigate the prevalence, the intensity and the relative frequency of multi-parasitism of the major soiltransmitted helminthes (Ascaris lumbricoides, Trichuris trichiura, and the hookworms) and Schistosoma mansoni. A cross sectional survey was conducted from February 10, 2011 up to May 10, 2011, in three primary schools in Libo-Kemkem District, northwest Ethiopia. 365(173 males and 192 females) pupils, selected using systematic sampling using a random start, had their fecal specimens been examined using Kato-Katz cellophane fecal thick smear technique (41.7 $\mathrm{mg}$ template). S. mansoni was the most prevalent (15.9\%) followed by A. lumbricoides (11.0\%), the hookworms (8.2\%) and T. trichiura (1.6\%) and the corresponding geometric mean egg counts per gram were 122, 1749, 582 and 13 respectively. Single, double and triple infections encountered were $21.1 \%, 4.9 \%$ and $1.9 \%$ respectively. Most of the double infections were a combination of $A$. lumbricoides and the hookworms (3.6\%). All schools combined, the average prevalence and egg count for each helminthes were neither age nor sex related but intensity of $S$. mansoni by age group which was statistically significant $(\mathrm{p}<0.05)$. The distribution of helminthes among pupils was over dispersed: that is, while most pupils harbored few or no and a few harbored many of them. Although, as compared to the previous studies, the present study showed a lowered infection rates, the relative increase in $S$. mansoni prevalence signifies a need for promoting intervention measures.
\end{abstract}

Keywords: Soil-transmitted helminthes infection, Schistosma mansoni, Ethiopia

\section{Background}

The burden of disease caused by infection with schistosomiasis and soil-transmitted helminths (STH) remains enormous [1]. About 2 billion people are affected worldwide, of whom 300 million suffer associated severe morbidity. World Health Organization (WHO) estimated that these infections represented more than $40 \%$ of the disease burden due to all tropical diseases, excluding malaria [1].

Among parasitic diseases in the tropics and subtropics, schistosomiasis ranks second to malaria in terms of socio- economic and public health importance, with an estimated 779 million people are at risk and 200 million infected $[2,3]$. An estimated $85 \%$ of all cases, and the majority of severe morbidity, are now concentrated in sub-Saharan Africa [3]. The yearly death rate in the region is estimated to be more than 200,000 [4]. Schistosomiasis has also long been recognized as a major public health problem in East Africa [5]. Ethiopia is one of the poorest and least developed countries in the world, endemic for many neglected tropical 
diseases (NTDs) including schistosomiasis [6].

The soil-transmitted helminths (STH): hookworms, $A$. lumbricoides and $T$. trichiura are also widely distributed in tropical countries [7], infecting 1300, 1450 and 1050 million people, respectively [8]

It is widely recognized that school children carry the heaviest burden of morbidity due to intestinal helminths and schistosomiasis infection [10].

Chronic inflammatory process associated with long-term schistosomiasis contributes to anemia and under-nutrition, which, in turn, can lead to growth stunting, poor school performance, poor work productivity, and continued poverty [9]. Hookworm infection is a public health problem of great magnitude and produces morbidity by blood loss, with consequent iron deficiency anemia and hypo-proteinaemia [10]. Ascariasis has an adverse effect on nutrition and also results in serious pathology due to migrations of the worms in the body. Trichuriasis is implicated in rectal prolapse and chronic dysentery [10]. Simultaneous and sequential transmission of multiple parasites, and their resultant overlapping chronic infections, are facts of life in many underdeveloped rural areas. These represent significant but often poorly measured health and economic burdens for affected populations [9].

Surveys carried out on Schistosomes and the geohelminths on school children in Ethiopia have shown that the helminthic infections were major public health problems in many areas [10]. Although few studies have tried to address the problems encountered by helminthes in certain communities in northwest Ethiopia, no study, to our knowledge, was conducted in the district of Libo-Kemkem. Because of this, the present study was conducted to determine the prevalence and the intensity of the major intestinal helminthes parasites and the relative frequency of multi-parasitism on school children.

\section{Methods and Materials}

\subsection{Study Design, Period and Area}

A cross-sectional survey using quantitative method was conducted. The study was carried out in Libo-kemkem district, South Gondar Zone; Northwest Ethiopia from February10, 2011 to May-10, 2011. The study area is located between Bahir Dar and Gondar on the major road connecting Addis Ababa to the Sudan.

\subsection{Study Population}

All schoolchildren in primary schools of the LiboKemkem district

\subsection{Variables}

Soil-transmitted helminths infections and Schistosomiasis mansoni was dependent variable. Age, sex, schools were independent variables.

\subsection{Sample Size and Sampling Procedures}

Sample size was calculated using single population proportion formula by taking the proportion of one or multiple parasitic infections from previous studies conducted in other part of the country i.e. $68.4 \%$ (10). By setting confidence interval at $95 \%$ and alpha at $5 \%$ and adding contingency of $10 \%$ a total of 365 sample size was calculated. The study was undertaken in three primary schools (Alabo, Addis Zemen and Abebaye primary School) out of those found in the District. Accessibility by four wheel drive and proximity for health centers was a factor in the selection of the schools to be involved in the study. Five sections were selected from each school by lottery method and the number of students to be included in the study from the selected sections of each school was determined based on proportion. The calculated proportion of students from sections of each school was selected by a systematic sampling technique using a random start from a complete register of five sections. Their ages and sexes were registered to a prepared format.

\subsection{Stool Collection and Examination}

Students were supplied with labeled plastic containers and applicator sticks to bring about $3 \mathrm{gms}$ of faeces. Samples collected from 365 students were processed with the KatoKatz cellophane thick smear technique using $41.7 \mathrm{mg}$ templates. Three slides were prepared for each sample and examined by well trained expertise. The eggs of all intestinal helminths encountered under the microscope (using the $10 \mathrm{x}$ eyepiece magnification) were counted and the average was taken when eggs found on the three slides. For each parasite species the average number of eggs counted was converted to eggs per gram of faeces by multiplying by a factor of 24 .

\subsection{Data Analysis}

Data was analyzed using SPSS version 16.0 and Stata SE 10 computer software and presented in tables and graphs. The Chi-square test and independent T-test were utilized to assess significant differences obtained in infection rates and egg counts among the age group and sex of schoolchildren under consideration. A difference was taken as significant at a p-value less than 0.05 .

\subsection{Quality Assurance}

The quality of the specimens was kept by giving orientation to the students how to collect before collection. The stool smear that was examined and said either positive or negative for any ova of helminthes was re-checked by other experienced person to decide its positivity or negativity.

\subsection{Ethical Clearance}

Ethical clearance was obtained from Institutional Review Board of Aklilu Lemma Institute of Pathobiology (ALIPB), Addis Ababa University and letter written by ALIPB to the Woreda Health and Education Office was taken to get permission to involve students in the study and get support 

among School Children in Libo-Kemkem District, Northwest Ethiopia: Cross Sectional Study

with materials respectively. The benefit of the study was explained to the directors of each school and students. Those students who were positive for any of the helminths were offered free treatment. Children positive for S. mansoni were treated on the spot with a single dose of praziquantel at $40 \mathrm{mg} / \mathrm{kg}$ stat body weight. However, those which were positive for the other helminthes were treated with single dose of albendazole $400 \mathrm{mg}$ stat body weight.

\section{Results}

A total of 365 children were examined. Of these 173 and 192 were males and females respectively (Table 1). The overall prevalence rate for one or multiple parasitic infections in the school children was $27.9 \%$ (table 3 ). Of the sampled school children, $15.9 \%, 11.0 \%, 8.2 \%$, and $1.6 \%$ had $S$. mansoni, A. lumbricoides, the hookworms and T. trichiura and the corresponding geometric mean egg counts per gram of faeces were 122, 1749, 582 and 13 respectively (table 1). The of the four helminthic infections was high in Alabo. $S$. mansoni was highest in prevalence in Alabo (20.3\%) followed by Abebaye (15.5\%) while the intensity of infection was highest in Alabo (144 epg) followed by Addis Zemen (112 epg). However, the intensity of A. Lumbricoides infection was highest (2064 epg) in Addis Zemen. Hookworm infection was most prevalent in Alabo (9.0\%) followed by Addis Zemen (8.6\%). The intensity of hookworm infection was highest (659 epg) in Abebaye where the prevalence was lowest $(6.9 \%)$ indicating that the few infected children could have high worm burdens. No $T$. trichuria infection was registered in Abebaye whereas the intensity of infection was comparable in Alabo and Addis Zemen (Table 1).

Table 1. Prevalence (\%) of intestinal helminthes infections and average egg counts per gram of faeces (EPG) including analysis by age group and sex in children attending three primary schools in Libo-Kemkem District, Northwest Ethiopia, February, 2011

\begin{tabular}{|c|c|c|c|c|c|c|c|c|c|c|c|c|c|}
\hline & \multirow{3}{*}{$\begin{array}{l}\text { No. } \\
\text { Exam }\end{array}$} & \multicolumn{12}{|c|}{ Positive for } \\
\hline & & \multicolumn{3}{|c|}{ S. mansoni } & \multicolumn{3}{|c|}{ A. lumbricoides } & \multicolumn{2}{|c|}{ Hookworms } & \multicolumn{4}{|c|}{ T. trichiura } \\
\hline & & $\#$ & $\%$ & GM* EPG & $\#$ & $\%$ & GM EPG & $\#$ & $\%$ & GM EPG & $\#$ & $\%$ & GM EPG \\
\hline \multicolumn{14}{|l|}{ Schools } \\
\hline Alabo P.S & 133 & 27 & 20.3 & 144 & 19 & 14.3 & 1611 & 12 & 9.0 & 613 & 4 & 3.0 & 13 \\
\hline Addis Zemen P.S & 116 & 13 & 11.2 & 112 & 11 & 9.5 & 2064 & 10 & 8.6 & 496 & 2 & 1.7 & 14 \\
\hline Abebaye P.S & 116 & 18 & 15.5 & 100 & 10 & 8.6 & 1702 & 8 & 6.9 & 659 & 0 & 0.0 & 0 \\
\hline \multicolumn{14}{|l|}{ Sex } \\
\hline Male & 173 & 33 & 19.1 & 101 & 22 & 12.7 & 2398 & 14 & 8.1 & 1013 & 2 & 1.2 & 8 \\
\hline Female & 192 & 25 & 13.0 & 156 & 18 & 9.4 & 1189 & 16 & 8.3 & 359 & 4 & 2.1 & 16 \\
\hline \multicolumn{14}{|l|}{ Age Groups } \\
\hline $5-9$ years & 192 & 36 & 18.8 & 147 & 21 & 10.9 & 1838 & 18 & 9.4 & 713 & 3 & 1.6 & 12 \\
\hline $10-14$ years & 173 & 22 & 12.7 & 89 & 19 & 11.0 & 1656 & 12 & 6.9 & 430 & 3 & 1.7 & 15 \\
\hline
\end{tabular}

*GM EPG=Geometric Mean Egg per Gram

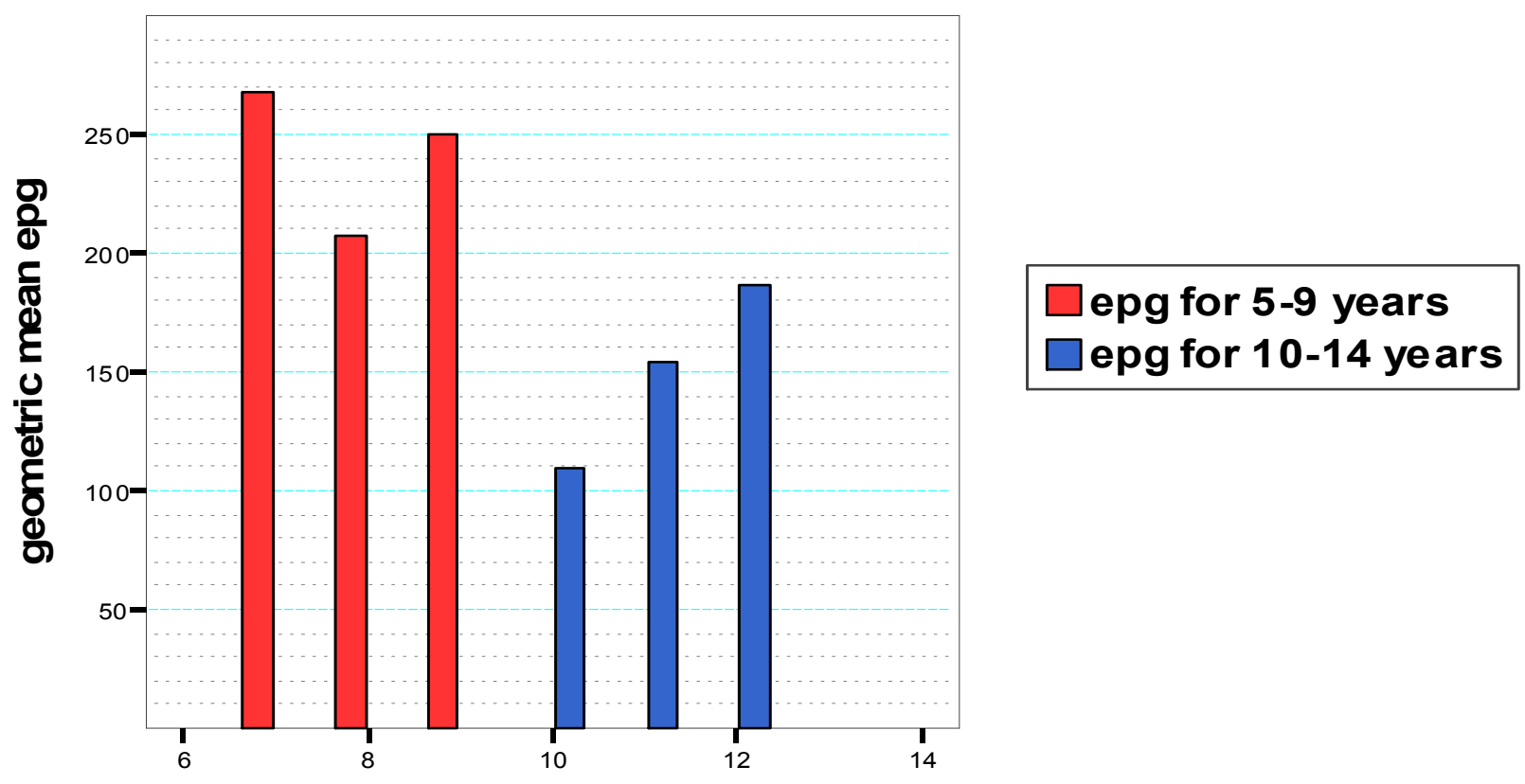

\section{Age in years}

Figure 1. Intensity of infection of S. mansoni by age group in children attending three primary schools in Libo-Kemkem District, Northwest Ethiopia, February, 2011 
No statistically significant difference was obtained in intensity of infection among the age groups and sexes of school children for A. lumbricoides, the hookworms and $T$. trichuria infections under consideration $(\mathrm{p}>0.05)$. This denotes a similar exposure risk to infection by these helminthes (table-1)

Analysis of age-specific intensities of $S$. mansoni infection showed that schoolchildren of the age group 5-9 years carried the heaviest load which was statistically significant $(\mathrm{p}<0.05)$ (Fig. 2) while no statistically significance difference was observed in prevalence $(\mathrm{P}>0.05)$ (table-1). Both prevalence and intensity of $S$. mansoni infection due to sex showed no statistically significant differences $(\mathrm{P}>0.05)$ (Table1).

The frequency distribution of egg counts of the helminthes among schoolchildren was over dispersed: i.e., while most children harbored few or no eggs, a few harbored many of them. This distribution has clinical consequences for the children, as it is mainly the intensity of infection that determines the severity of morbidity. But for T. trichuria, the prevalence of both moderate and heavy infections was zero (table 2).

Table 2. Classification of intensity of infection of $S$. mansoni, A. lumbricoides, T. trichiura, and the hookworms in children attending three elementary schools in Libo Kemkem District, Northwest Ethiopia, February, 2011

\begin{tabular}{lll}
\hline \multirow{2}{*}{ Egg range } & All school & \\
\cline { 2 - 3 } & No. exam. & $\%$ \\
\hline S. mansoni & & \\
Negative (0 epg) & 307 & 84.1 \\
Light infection (1-99 epg) & 26 & 7.1 \\
Moderate infection (100-399 epg) & 26 & 7.1 \\
Heavy infection (>400 epg) & 6 & 1.6 \\
Total & 365 & 100.0 \\
A. lumbricoides & & \\
negative (0 epg) & 325 & 89.0 \\
light infection (1-4999 epg) & 30 & 8.2 \\
moderate infection (5000-49999 epg) & 8 & 2.2 \\
heavy infection (>50000 epg) & 2 & 0.6 \\
Total & 365 & 100.0 \\
Hookworms & & \\
negative (0 epg) & 335 & 91.8 \\
light infection( $1-1999$ epg) & 27 & 7.4 \\
moderate infection (2000-3999 epg) & 1 & 0.3 \\
heavy infection ( $>4000$ epg) & 2 & 0.5 \\
Total & 365 & 100.0 \\
T. trichuria & & \\
Negative (0 epg) & 359 & 98.4 \\
Light infection (1-999 epg) & 6 & 1.6 \\
T0tal & 365 & 100.0 \\
\hline
\end{tabular}

Triple, double and single infections were found in $7(1.9 \%)$, $18(4.9 \%)$ and $77(21.1 \%)$ of stool specimens, respectively. Most of the double infections were a combination of $A$. lumbricoides and the hookworms 13(3.6\%). Only S. mansoni, A. lumbricoides, and the hookworms occurred as triple infection while no quadruple infection was recorded. This may be due to a decrease in prevalence of infections. The highest prevalence for a single infection was recorded for $S$. mansoni 46(12.6\%) followed by A. Lumbricoides 18 (4.9\%) (Table 3).
Table 3. Multiple intestinal helminthic infections in children attending three elementary schools in Libo Kemkem District, northwest Ethiopia, 2011

\begin{tabular}{lllllll}
\hline Multiplicity of & Males & \multicolumn{3}{c}{ Females } & \multicolumn{2}{c}{ Both sexes } \\
\cline { 2 - 7 } infections & $\#$ & \% & $\#$ & \% & $\#$ & \% \\
\hline $\begin{array}{l}\text { Specimens with 3 } \\
\text { helminth parasites }\end{array}$ & & & & & & \\
SM. AL, and HW & 5 & 2.9 & 2 & 1.0 & 7 & 1.9 \\
$\begin{array}{l}\text { Specimens with 2 } \\
\text { helminth parasites }\end{array}$ & & & & & & \\
SM and AL & 0 & 0.0 & 2 & 1.0 & 2 & 0.5 \\
AL and HW & 6 & 3.5 & 7 & 3.6 & 13 & 3.6 \\
SM and HW & 1 & 0.6 & 0 & 0.0 & 1 & 0.3 \\
SM and TT & 1 & 0.6 & 1 & 0.5 & 2 & 0.5 \\
Specimens with 1 & & & & & & \\
helminth parasite & & & & & & \\
SM & 26 & 15.0 & 20 & 10.4 & 46 & 12.6 \\
AL & 11 & 6.4 & 7 & 3.6 & 18 & 4.9 \\
HW & 2 & 1.2 & 7 & 3.6 & 9 & 2.5 \\
TT & 1 & 0.6 & 3 & 1.6 & 4 & 1.1 \\
Total Positive & 53 & 30.6 & 49 & 25.5 & 102 & 27.9 \\
Total Negative & 120 & 69.4 & 143 & 74.5 & 263 & 72.1 \\
Total & 173 & 100.0 & 192 & 100.0 & 365 & 100.0 \\
\hline
\end{tabular}

$\mathrm{SM}=\mathrm{S}$. mansoni, $\mathrm{HW}=$ Hookworm, $\mathrm{AL}=\mathrm{A}$. lumbricoides, $\mathrm{TT}=\mathrm{T}$.trichiura

\section{Discussion and Conclusion}

The main interests of this study were prevalence, intensity and multiplicity of major intestinal helminthes infection. According to the result of this project the prevalence of $S$. mansoni, A. lumbricoides, the hookworms and T. trichiura infections among school children were $15.9 \%, 11.0 \%, 8.2 \%$ and $1.6 \%$ respectively. This result is larger as compared to the study conducted in the semi-pastoralist tribes in lower Omo Valley [11]. This difference may be attributed to the difference in environmental conditions; hence arid condition is not favorable for larval development in previous study.

Surveys conducted by Jemaneh on intestinal helminths among school children in Chilga District, North Gondar Zone reported overall prevalence's of $19.4 \%, 42.9 \%, 37.7 \%$, and $14.8 \%$ for S. mansoni, A. lumbricoides, hookworm and $T$. trichira infections respectively which are higher than our report [10]. Environmental sanitation, water supply and socioeconomic status of households may be contributing factors for the difference, although this needs to be verified in more extensive follow up studies. Other factors related to macro-and micro-environment, time of study, method of examination, etc., do also play a role.

Higher results were recorded as compared to the present study on researches conducted on intestinal parasites among communities in different parts of Ethiopia [12]; may be resulting from difference in methodologies used; because less sensitive technique was used in this study than the previous. On the other hand, a report from a study on intestinal helminthes among residents of the Finchia Sugar Plantation Area showed a higher prevalence than the current study [13]. The previous project area can be more favorable for 
development, survival and transmission of helminths. Among school children, S. mansoni (21.2\%), A. lumbricoides $(6.2 \%)$, the hookworms $(60.2 \%)$ and T. trichuria $(14.7 \%)$ infections were reported [14]. Results were higher but for $A$. lumbricoides than this project; implication of the sampling differences.

In General, compared to the previous reports, the present study showed lower prevalence of the helminthes infection. This may be due to an increase in people's awareness on personal hygiene, environmental sanitation, use of latrines created by health extension workers in a rural area and weekly health package programs with other health workers in Urban. However, the prevalence obtained still indicates as more effort has been anticipated from health extension workers and other health professionals to create awareness on the epidemiology and controlling methods of intestinal helminthes in the community, schoolchildren in particular.

In school children of Babile town, a prevalence of $4.3 \%$ for $S$. mansoni and $6.7 \%$ for the hookworms was registered [15]. The prevalence of the hookworm is comparable with the present project but that of $S$. mansoni is much lower which is an implication of a frequent exposure of children to schisto-infested water in the current project Area. A report from Nigeria, Ecuador and Malawi showed a higher prevalence among school children than this study $[16,17,18]$ The difference may arise due the difference in the study communities; i.e. immunity, feeding habit, living condition and awareness on health.

No significant difference was obtained in infection rates and egg counts among the age group and sex of school children under consideration but $S$. mansoni intensity of infection by age group. This denotes more or less a similar exposure risk to infection by these helminths.

The intensity of helminthic infection in this study has been assessed indirectly by egg counts in faeces. This method, although susceptible to errors of sampling due to periodicity of egg production by female worms which may lead to uneven distribution of eggs in faeces, is still widely used as a measure of intensity of intestinal helminthic infections [19). Using this method, study on the intensity of infection has been carried out among school children of Dembia plains in North Gondar area. The intensity of infection was found to be comparable with the present study [20]. Similar patterns of intensity of infection have been observed elsewhere [13]. But much higher EPGs for the helminths was observed in a study conducted in Adarkay District than that of the present project [21]. The difference may be due to different factors; i.e. environmental sanitation, water supply, socio-economic status, immunity, and differences in exposure to infection.

The categorization of the intensity of infection due to $S$. mansoni, A. lumbricoides, T. trichiura, and the hookworms showed that majority of the sampled children were negative or few egg excreters. But, a few produced moderate and heavy eggs. Similar findings have been reported from a study conducted in the Fincha Sugar Plantation area [13]. Reverse findings were found in Chilga Districts and Dembia plains in Ethiopia and in Nigeria also heavy infection was higher [21,
$20,16]$. This may be attributed to the range of values used for classification; that means in the present study the WHO standard is used. The high degree of aggregation of eggs in the infected population has an implication in the contamination of the environment.

Multiple helminthes infection is a common phenomenon in areas where different types of parasites are encountered. In double infection, $S$. mansoni and $A$. lumbricoides combinations were most common in the study conducted in Chilga District similar to the present study while different in triple infection combination [21]. In triple infection, combination of $S$. mansoni, A. lumbricoides and the hookworms were most common in this study, similar to the previous reports in Dembia Plains and Adarkay but different in double infection Combination [20].

This is probably due to the higher prevalence of the three helminths in the study communities. In conclusion, investigating the prevalence, the intensity and multiparasitism of intestinal helminthes, S. mansoni and major STH in particular, in school children is of paramount importance in illustrating their health status and the problems that need to be addressed to improve their health and wellbeing. Even though, the overall prevalence, in this study, was lower as compared to most of the previous reports in different areas; still the predominant prevalence of $S$. mansoni, followed by $A$. lumbricoides and the hookworms along with the heaviest burdens raises a serious concern. As helminthes infection and disease adversely affect child growth and development, nutritional status and cognitive capacity which have been proportionally associated with worm burden, intervention measures i.e. health education on personal hygiene, controlling mechanisms and ways of transmissions of helminths infections, proper use of latrines, consequences of open field defecation and other healthpromoting behavior should target school children, with long term improvements of sanitation and provision of clean water to the community.

\section{Strength and Weakness}

\subsection{Strength}

The results of stool examination was re-checked by more experienced laboratory personnel and as much as possible appropriate stool examination method is used for its maximum quality

\subsection{Weakness}

Like most other health studies, data from cross-sectional studies, by its nature has a defect to detect cause and effect relationship; there could be social desirability and recall bias by respondents to address all relevant variables and since the study did not include associated factors, the relationship with contributing factors could not be explained. The sampling method used may affect the validity of the result but we use it due to resource constraint 


\section{Authors' Contribution}

TA: Conceptualized the research problem, designed the study, prepared the proposal, conducted field work, and analyzed the data, manuscript writing.

AA: Participated during analysis of data, preparing the manuscript for publication

\section{Acknowledgements}

We would like to express our grateful heartfelt appreciation to Libo-kemkem district Education Office and School Administrators, data collectors and supervisors of the study for their cooperation and support, giving unreserved time and follow up from the beginning till completion of this research. Our acknowledgement also extends to study participants who were involved in this study.

\section{References}

[1] World Health Organization (WHO): Schistosomiasis and soiltransmitted helminth infections - preliminary estimates of the number of children treated with Albendazole or mebendazole: Weekly epidemiological record. Geneva, 2006, 81: pp 145164

[2] Ali AS. Natural products as therapeutic agents for schistosomiasis. Research Journal of Medicinal Plants, 2011, 5(1): pp 1-20

[3] WHO. Schistosomiasis and intestinal parasites control planning and technical guidance communicable diseases prevention and control: Report of the WHO informal consultation on schistosomiasis control. WHO/CDS/CPC/SIP/99.2, GENEVA,WHO. 1998

[4] Van derWerf MJ, De Vlas SJ, Brooker S, Looman CWN, Nagelkerke NJD, Habbema JDF, et al. Quantification of clinical morbidity associated with Schistosoma infection in Sub-Saharan Africa, Acta Trop, 2003, 86: pp 125-39

[5] Malone JB, Yilma JM, McCarroll JC, Erko B, Mukaratirwa S, Zhou X. Satellite climatology and the environmental risk of Schistosoma mansoni in Ethiopia and East Africa, Acta Trop, 2001, 79: pp 59-72

[6] Tadesse Z, Hailemariam A, Kolaczinskic JH. Potential for integrated control of neglected tropical diseases in Ethiopia: mini-review. Trans R Soc Trop Med and Hyg, 2008, 102: pp 213-214

[7] Mwangi TW, Bethony J, Brooker S. Malaria and helminths interactions in humans: an epidemiological viewpoint. Ann Trop Med Parasitol, 2006, 100: pp 551-570

[8] Montresor A, Crompton DWT, Gyorkos TW, Savioli L. Control Strategies-helminth control in school-aged children: a guide for managers of control programmes, WHO, 2002
[9] King HC. Parasites and poverty-the case of schistosomiasis: review, Acta Tropica, 2010, 113: pp 95-104

[10] Jemaneh L. Soil-transmitted helminth infections and Schistosomiasis mansoni in school children from Chilga District, Northwest Ethiopia. Ethiop J Health Sci, 2001, 11: pp $79-87$

[11] Tilahun T. Intestinal parasitosis among Kara and Kwego emipastoralist tribes in lower Omo Valley, Southwestern Ethiopia. Ethiop J Health Dev, 2009, 23: pp 57-62

[12] Assefa T, Woldemichael T, Dejene A. Intestinal parasitism among students in three localities in South Wello, Ethiopia. Ethiop J Health Dev, 1998, 12(3): pp 231-235

[13] Birrie H, Medhin G, Erko B, Beshah G, Gemetchu T. Intestinal helminthes infections among the current residents of the future Finchaa Sugar Plantation Area, Western Ethiopia, Ethiop J Health Dev, 1997, 11: pp 219-228

[14] Legesse $M$ and Erko B. Prevalence of intestinal parasites among school children in a rural area close to the Southeast of Lake Langano, Ethiopia, Ethiop J Health Dev, 2004, 18: pp $116-120$

[15] Tadesse G. The prevalence of intestinal helminthic infections and associated risk factors among school children in Babile town, Eastern Ethiopia, Ethiop J Health Dev, 2005, 19(2): pp $140-147$

[16] Andy EO and Palmer AD. Soil-transmitted helminthiasis among school age children in Ethiope East Local Government Area, Delta State, Nigeria, African Journal of Biotechnology, 2005, 4: pp 938-941

[17] Jacobsen KH, Ribeiro PS, Quist BK, and Rydbeck BV. Prevalence of intestinal parasites in Young Quichua Children in the Highlands of Rural Ecuador, J Health Popul Nutr, 2007, 25: pp 399-405

[18] Randal PAE, Perez MA, Floyd S, Black GF, Crampin AC, Ngwira B, Piston WN, Mulawa D, Sinhala L, Mwaungulu L, Bickle Q, Fine PEM. Patterns of helminth infection and relationship to BCG vaccination in Karonga District, Northern Malawi, Trans R Soc Trop Med and Hyg, 2002, 6: pp 29-33

[19] Croll NA, Anderson RM, Gyorkos TW, Ghadirian EA. The population biology and control of Ascaris lumbricoides in a rural community in Iran, Trans Roy Soc Trop Med Hyg, 1982, 76: pp 187-97

[20] Jemaneh L. Schistosomiasis mansoni and geo-helminthiasis in school children in the Dembia plains, Northwest Ethiopia, Ethiop J Health Dev, 1998, 12(3): pp 237-244

[21] Jemaneh L. Intestinal helminths infections in school children in Adarkay District, Northwest Ethiopia, with special reference to Schistosomiasis mansoni, Ethiop J Health Dev, 1997, 11(3): pp 289-294 